\title{
Archives orales et entretiens ethnographiques: un débat entre Florence Descamps et Florence Weber
}

\author{
animé par Bertrand Müller*
}

L'entretien constitue aujourd'hui l'un des instruments privilégiés des sciences sociales. Mais c'est peut-être moins l'oralité qui en a fait le succès que la relation particulière entre enquêteur et enquêté qui en explique la promotion. L'entretien est en effet plus qu'un simple dialogue entre un questionneur et un questionné, il est bien un rapport social et verbal configuré dans un moment et un espace singuliers pendant et dans lequel, tel en est à la fois l'enjeu et le pari, se jouent une interaction non contrainte qui modifie l'information produite. Cette interaction repose sur une relation contractuelle dans laquelle l'enquêteur et l'enquêté ont pu définir à l'avance la nature même de leur relation qui n'est ni une interrogation, ni non plus un échange clinique ou thérapeutique, ni même un simple jeu de questions réponses comme c'est le cas dans les entretiens journalistiques.

La relation d'entretien se différencie également des grandes enquêtes sociales qui avaient été pratiquées dans leur début par des fonctionnaires, mais aussi des médecins, des prêtres se situant ainsi à la charnière des dominants et des dominés reproduisant à la fois l'écart social et cognitif qui séparait les enquêteurs des enquêtés. Le dispositif demeurait celui de l'interrogatoire et le protocole celui de la visite. L'enquêteur, porte-parole des autorités sociales ou intellectuelles dont il est le médiateur

* Département d'Histoire Générale, Université de Genève. 
et le représentant, administre un questionnaire sur des thèmes qui préoccupent les autorités sans se préoccuper des enquêtés qui sont souvent des dominés. L'entretien propose une perspective nouvelle sensible déjà chez Le Play lorsqu'il affirmait: "Mieux vaut écouter qu’interroger.

L'entretien entend ainsi opérer un double déplacement: redresser la relation inégale entre observateur et observé et libérer la parole par rapport à l'écrit en offrant la possibilité à ceux qui n'en ont ni l'autorité ni la légitimité de "prendre la parole". Ces perspectives ont sans doute largement idéalisé l'entretien dans certaines disciplines comme l'histoire contemporaine qui se cherchait une légitimité nouvelle en introduisant l'oral, le récit de vie, pour rompre avec l'impérialisme de l'écrit. Paradoxalement, dans d'autres disciplines, longtemps dominées par l'oralité avant d'être saisies elles aussi par l'archive, comme l'anthropologie ou l'ethnographie, l'entretien n'a pas nécessairement été considéré comme un instrument privilégié.

Dans une revue comme la nôtre, préoccupée de confronter l'interdisciplinarité sur le terrain des pratiques, le débat sur les méthodologies transdisciplinaires figurent à l'évidence parmi nos préoccupations majeures et nous avons publié régulièrement des articles recourrant aux méthodes de l'entretien. Cependant, nous avons souvent souhaité pouvoir confronter directement des pratiques disciplinaires différentes sur les enjeux de l'entretien. Le débat que nous vous proposons entre une ethnographe, Florence Weber, et une historienne, Florence Descamps, débat qui s'est tenu lors de la journée annuelle du Laboratoire de sciences sociales de l'Ecole normale supérieure en juin 2005, présente très concrètement les enjeux méthodologiques et épistémologiques liés à des usages qui se révèlent très différents entre les deux disciplines. Pour ce débat, nous avons retenu trois chapitres, nous proposant dans un premier temps de replacer l'entretien dans l'histoire des deux disciplines, puis de situer mieux son usage dans des stratégies de recherches elles aussi différenciées, enfin nous avons voulu aborder la question des enjeux patrimoniaux et archivistiques de l'entretien non seulement pour les historiens qui privilégient aujourd'hui l'archive orale plutôt que l'histoire orale mais aussi pour les ethnologues.

B.M. 
Florence Descamps: Ancienne élève de l'Ecole normale supérieure et agrégée d'histoire, Florence Descamps est maître de conférence en histoire à l'Ecole Pratique des Hautes Etudes (EPHE). Elle a collaboré ou dirigé plusieurs programmes ministériels d'archives orales, notamment sur l'histoire de l'Etat depuis l'entre-deux-guerres (Economie et Finances, Culture, Affaires Sociales, Intérieur etc.). Elle anime un séminaire de méthode à l'EPHE sur la constitution de la source orale et son utilisation dans les sciences sociales, ainsi que sur le patrimoine oral en France au xx siècle. Elle poursuit par ailleurs des recherches sur l'histoire du ministère des Finances au xx ${ }^{\mathrm{e}}$ siècle. Elle a publié notamment: Descamps Florence. 2001. L'bistorien, l'archiviste et le magnétophone. De la constitution de la source orale à son exploitation. Paris, Comité pour l'Histoire Economique et Financière (CHEFF), réed. 2006.

Florence Weber: Anthropologue de formation, Florence Weber enseigne la sociologie et l'ethnographie à l'Ecole normale supérieure $(\mathrm{Pa}$ ris) depuis 1983. Aujourd'hui chercheur au Centre d'études de l'Emploi, elle a commencé sa carrière de chercheur à l'INRA et au Laboratoire de Sciences sociales de l'ENS qu'elle a dirigé de 2002 à 2005. Membre depuis 1990 du comité de rédaction de la revue Genèses. Sciences sociales et histoire, où elle a accompagné la publication de nombreux travaux ethnographiques et la réflexion sur les méthodes des sciences sociales, elle a publié avec Stéphane Beaud le Guide de l'Enquête de terrain, La Découverte (1997, édition augmentée 2003). Membre du comité de rédaction de la revue Ethnography, elle défend une conception de l'ethnographie sociologique proche de ce que les anglophones appellent aujourd'hui reflexive ethnography: utiliser les méthodes classiques de l'anthropologie (observation participante) et de la sociologie (entretien), soumises aux rigueurs de l'auto-analyse, appuyées sur une théorie qui combine analyse des interactions et analyse des institutions.

\section{L'entretien entre histoire orale et ethnologie}

Bertrand Müller: Pour entrer en matière, je vous demande simplement de préciser et de situer respectivement, l'histoire orale et l'entretien dans vos disciplines de référence, qui sont d'une part l'histoire, d'autre part l'ethnologie. 
Florence Descamps: Chronologiquement, on peut distinguer quatre périodes. 1) Les années 1890-1930 organisent un bannissement de la source orale. 2) Les années 1930-1960 voient se produire un lent ébranlement de la suprématie du document d'archive. 3) 1960 à 1980 correspond à l'acclimatation de la pratique de l'entretien en histoire et suscite un certain nombre de débats méthodologiques: c'est le temps de l'histoire orale. 4) A partir des années 1990, à l'occasion du tournant mémoriel et patrimonial, s'effectue une sorte d'académisation et de banalisation de la pratique de l'entretien en histoire contemporaine qui se traduit par la mise en place des archives orales et la constitution de corpus de témoignages oraux rationalisés.

Je pense qu'il n'y a pas de continuité, même si les historiens de l'Antiquité grecque et chrétienne ont théorisé précocement la figure du témoin. L'entretien est une réelle innovation qui s'inscrit dans un contexte disciplinaire qui jusque-là récusait la source orale: l'histoire ne devait se faire qu'à partir des documents écrits et essentiellement des archives publiques. Bien sûr, grâce aux Annales notamment, il y a eu un début de desserrement de cet étau dans les années trente ; les historiens ont annoncé, de façon programmatique, une diversification des sources: archéologiques, cartographiques, iconographiques, photographiques, littéraires etc. Mais cette tendance ne s'est épanouie que tardivement. La réflexion sur la pratique de l'entretien n'a émergé que dans les années 1970, sous plusieurs influences, celle de l'oral history américaine ou européenne, et celle de la sociologie qualitative et compréhensive américaine puis française.

Il existe un fil directeur continu dans la discipline historique depuis l'Antiquité, qui est la réflexion sur le témoignage. Au xx siècle, en France, par exemple, il y a eu notamment un moment important au début des années trente, lorsqu'une discussion s'est engagée autour du travail de Jean Norton Cru qui avait décidé de collecter les récits de guerre publiés, de les critiquer et de les classer selon leur degré de véracité et de scientificité (Cru, 1993). Cette réflexion sur le témoignage individuel a été partagée par Marc Bloch qui a lui aussi réfléchi à la psychologie du témoignage (Bloch, 1922) et à son utilisation par l'historien ; il a d'ailleurs pensé collecter les "rapports" des anciens combattants pour faire l'histoire de la guerre dans les tranchées. Le recueil des témoignages des soldats de la Première Guerre mondiale ne se fera pas, mais il se fera après la Deuxième Guerre mondiale, grâce à un Comité d'histoire qui mènera plus de 2000 
entretiens auprès des résistants, des prisonniers et des déportés. Cette intuition pionnière sera reprise dans les années 1970 par l'organisation d'auditions enregistrées de "grands" témoins de la guerre, de l'Occupation et de la reconstruction, apportant ainsi une pierre à l'histoire orale naissante.

L'histoire orale est donc à la fois le produit d'une innovation réelle, pas totalement acceptée ni assumée aujourd'hui encore, et d'une réflexion proprement historique sur la notion du témoignage écrit ou oral ; ce qui marque à la fois la proximité et la frontière avec les autres sciences sociales.

Florence Weber: Pour l'ethnographie, la situation est assez contrastée. Il n'y a pas de consensus sur la question de l'entretien. L'ethnographie n'est pas une discipline mais une méthode au croisement de deux disciplines, la sociologie et l'anthropologie sociale (ou ethnologie). L'anthropologie sociale classique des sociétés sans écriture n'a jamais eu de véritable pratique de l'entretien ; elle était fondée sur l'observation, la prise de notes, le recensement. Les entretiens ne servaient que dans des cas particuliers: l'étude de la langue, celle des mythes. Lorsque les anthropologues se sont tournés vers l'étude des sociétés contemporaines, c'està-dire connaissant l'écriture et l'Etat, ils ont utilisé un peu plus systématiquement l'entretien, technique empruntée à la psychologie sociale et à la sociologie qualitative. Mais sans enthousiasme. En sociologie, la technique de l'entretien enregistré, long et non directif, s'est imposée sous le nom de "sociologie qualitative", en opposition à la sociologie quantitative qui raffinait ses techniques statistiques sans trop s'interroger sur les conditions de production de ses données. Mais cette sociologie qualitative restait fascinée par la quantité: quantité d'entretiens retranscrits, comptage des mots grâce aux statistiques textuelles. Ces excès et ce manque de réflexivité a conduit une partie des ethnographes, qu'ils soient anthropologues ou sociologues, à refuser l'usage des entretiens et à donner un primat épistémologique à l'observation. Les enquêtés ne sauraient pas ce qu'ils font et on ne devrait pas accorder le moindre crédit à leur discours.

L'obsession des entretiens retranscrits tout comme la méfiance envers la parole des enquêtés ont largement laissé la place aujourd'hui à une attitude à la fois plus nuancée et plus rigoureuse: la combinaison systématique entre observation des pratiques, écoute attentive (proche de la sociolinguistique) et, le cas échéant, enregistrement des discours, et objectivation des positions sociales. C'est cette ethnographie sociologique que nous avons enseignée ici, Stéphane Beaud et moi-même. La méthode s'est 
forgée, dès l'origine, dans les institutions où se rencontraient la tradition anthropologique et la tradition sociologique: la première école de Chicago, avec l'anthropologue Robert Redfield et les sociologues Robert Ezra Park et Ernest W. Burgess, l'école anthropologique anglaise d'inspiration durkheimienne, derrière Bronislaw Malinowski, en France plus modestement l'ethnographie métropolitaine au Musée des Arts et Traditions Populaires avec Marcel Maget puis l'école de Pierre Bourdieu. Cette ethnographie sociologique ne dissocie pas l'observation des pratiques au cours d'une enquête longue, comme les anthropologues, et l'entretien enregistré, qu'on a appelé "entretien ethnographique" pour le distinguer des entretiens accumulés et décontextualisés par la sociologie qualitative.

Il me semble qu'en France, l'histoire assez heurtée et marginale de cette ethnographie sociologique, qui s'est tenue à distance de la psychologie sociale et de la sociologie qualitative, croise deux fois celle des archives orales, au moment des deux guerres mondiales. Pendant la Première Guerre mondiale, où meurt Robert Hertz, sociologue durkheimien qui aurait pu donner ses lettres de noblesse à l'ethnographie française, le travail de Marc Bloch sur le témoignage s'effectue en lien avec celui des folkloristes, eux-mêmes liés à ce qu'on appelait alors la "psychologie collective". Après la Deuxième Guerre mondiale, le folklore meurt comme discipline scientifique ; son principal représentant français, un élève de l'archéologue durkheimien Henri Hubert, André Varagnac, dont le rôle pendant le régime de Vichy suscite de nombreuses interrogations, a légué sa méthode de travail au Comité d'histoire de la Deuxième Guerre mondiale, et ses intérêts de recherche à l'ethnographie des sociétés européennes, par l'intermédiaire de Marcel Maget, qui rompt dès 1946 avec l'accumulation de données où s'étaient enlisés les folkloristes.

L'ethnographe aujourd'hui doit son journal de terrain et ses fiches à la tradition anthropologique, il doit son magnétophone à la sociologie qualitative.

BM: Je voudrais demander une précision à Florence Descamps sur la question de la source et de l'histoire orales. On ne peut pas limiter l'histoire orale à la collecte de sources: dans les années 1970, l'histoire orale se développe autour de la question: comment faire de l'histoire sociale de catégories sociales qui n'ont pas de sources et dont le passé n'est pas trop lointain? Il y a donc là une démarche que l'on peut rapprocher de l'ethnographie et qui n'a pas vocation à s'inscrire dans une dimension patrimoniale. Il s'agit 
bien de produire de la connaissance mais aussi de développer certaines formes de récits plus proches des "interviewés" qui sont principalement des dominés ou des marginaux.

FD: En effet, cela a été une ambition de l'histoire orale de donner une place à une histoire des dominés. L'histoire des gouvernants est faite grâce aux archives écrites, l'idée était de créer de nouvelles sources pour saisir la vie, les représentations et l'histoire des dominés. La difficulté est venue de ce que les partisans de cette nouvelle approche lui ont donné le nom d'histoire orale, ce qui a contribué à raidir les tenants de la discipline qui ont rétorqué: "Mais ces récits de vie ne sont pas de l'histoire, ce sont uniquement des matériaux, des données, qu'il faut critiquer et retravailler pour construire un discours proprement historique." Ce fut notamment le combat de Dominique Aron-Schnapper qui a dit: "Arrêtons de parler d'histoire orale. De même qu'il y a des archives écrites, il faut parler d'archives orales, qui seront assignées ensuite en tant que sources orales par la réflexion spécifique de l'historien.” La querelle sémantique à cet égard a été très importante, elle a été une querelle de posture et d'institution, mais elle a concerné aussi les pratiques. Puis, on a dépassé l'affrontement entre histoire orale et archives orales pour déboucher sur un terme qui met à peu près tout le monde d'accord: les sources orales. On utilise aussi le terme de "témoignages oraux", qui gomme la dimension patrimoniale mais met l'accent sur l'implication du sujet parlant et sur son discours subjectif. Dans tous les cas, c'est l'historien en dernier ressort qui, par sa réflexion, constitue ses différents matériaux, écrits ou oraux, en sources, à partir desquelles il construit une connaissance du passé.

Aujourd'hui, les archives orales, les sources orales, font partie de la boîte à outil de l'historien, mais à condition qu'il réfléchisse sur la construction de ses sources. La question centrale est donc celle du contexte de production des matériaux et des données en histoire.

FW: Pour rebondir sur ce sujet, il faut dire que l'ethnographe a mis longtemps avant de réfléchir sur la construction de ses matériaux. Il n'a pas encore vraiment admis que ses matériaux puissent être réutilisés par d'autres. La délégation du travail de terrain reste relativement taboue, l'intérêt et la possibilité de réutiliser des matériaux constitués dans le cadre d'une enquête antérieure reste en discussion. Cela se fait aux Etats-Unis, mais en France il y a de fortes résistances à l'idée que l'ethnographe puisse se détacher des matériaux qu'il a produit. 
FD: De ce point de vue, l'histoire paraît en avance. En premier lieu, il me semble que la discipline entretient un rapport relativement distancié vis-à-vis de ses sources. Elle sait que les archives sont construites et traduisent un certain point de vue (institutionnel, individuel). Si elle s'inscrit dans une démarche plus quantitative, elle sait qu'elle va devoir construire des données statistiques, et les historiens oraux savent qu'ils construisent une source de façon artificielle. Cela est acquis depuis la fin des années 1980-1990. Bien sûr, chez l'historien oral, il peut exister une relation fortement "propriétaire" à ses sources, mais il me semble que progresse actuellement l'idée que les entretiens peuvent devenir "archives orales" pour la collectivité, que les témoignages appartiennent également aux témoins et finalement, que les sources orales constituées à un moment t pourraient avoir plusieurs vies.

Secundo, à partir du moment où il a été admis que les corpus oraux pouvaient être déposés dans des institutions publiques, c'est-à-dire patrimonialisés, la question de leur réemploi ne pouvait qu'aller de soi. Une réflexion importante, méthodologique, juridique mais aussi éthique, s'est d'ailleurs développée autour des protocoles de dépôt et des règles de communicabilité, sur la manière de réutiliser les corpus de témoignages déposés.

BM: On va un peu vite, on est passé au point trois. De l'idée qui animait les historiens à propos de catégories sociales "sans archives", nous sommes passés à une autre ambition, celle de recueillir des informations supplémentaires. Complémentaires aux sources écrites, comme s'il fallait, et/ou était possible de combler les lacunes de l'archive écrite. Comme si la production documentaire d'un temps, en l'occurrence le nôtre, demeurait insuffisante pour en écrire l'histoire.

FD: Il s'est produit en effet un déplacement entre l'idée de donner la parole à des gens qui en étaient privés à une toute autre discussion, plus disciplinaire, plus méthodologique, plus épistémologique consistant à faire la critique des outils de connaissance de l'histoire et à en élargir la panoplie, de manière à pouvoir saisir le réel ou la société de manière plus ajustée et plus fine. De même que la source archéologique ou photographique permet d'atteindre d'autres objets, la source orale doit permettre d'atteindre d'autres objets. Après l'idée de rendre la parole aux "petits" et de la recherche-action, aujourd'hui ce qui domine, c'est l'allongement et l'affinement du questionnement de l'historien. 
BM: Du côté de l'ethnographe, les résistances paraissent plus fortes à l'idée d'un usage autonome de la source.

FW: Pour un ethnographe, la plupart des termes que vous employez depuis un moment n'ont aucun sens: collection de témoignages oraux, corpus, et même "témoignage". Ce qui est important pour nous, c'est que l'enquête ethnographique ne peut pas être conçue en dehors des interactions entre l'enquêteur et l'univers social où son intervention, sa présence vont changer les choses. Ces changements vont précisément lui apprendre ce qu'est cet univers social. A partir de là, l'idée de l'entretien comme texte devient problématique. Il nous arrive d'utiliser des textes d'entretiens, bien sûr - nous réfléchissons à ce qu'est la transcription -, mais d'un point de vue ethnographique, on ne peut pas isoler un entretien de l'ensemble de l'enquête conçue comme déroulement significatif d'interactions entre un enquêteur (rarement plusieurs) et des enquêtés. On aura donc une grosse difficulté à penser les entretiens comme une collection de témoignages, c'est-à-dire comme des textes autonomes, décontextualisés: ce ne sont pas des textes mais des transcriptions d'interactions, prises dans une histoire, celle de l'enquête, plus importante parfois que ce qui s'y est dit. C'est un peu le même problème que l'on a avec les statistiques. L'ethnographe a beaucoup de mal à penser statistiquement c'est-àdire à mettre ensemble des données en laissant de côté la série des interactions qui les ont produites. Nous avons du mal à utiliser des données extraites de leur contexte, nous sommes trop attentifs à la construction de nos propres données. Nous n'avons pas d'intérêt pour la donnée plate, séparée de ses conditions de production.

Il y a un autre élément intéressant. L'anthropologie a connu presque un mouvement inverse par rapport à ce que vous décrivez: c'est aujourd'hui que se développe, aux Etats-Unis et en Australie principalement, le mouvement qui veut rendre la parole aux indigènes. Ailleurs ce mouvement a été paradoxalement peu important même après la décolonisation, mais aujourd'hui la question revient avec ce qu'on pourrait nommer l'émergence d'une anthropologie indigène, une anthropologie faite par des anthropologues issus des sociétés indigènes.

La décolonisation avait arrêté les anthropologues dans le développement de leur discipline et marqué le repli de l'anthropologie des anciennes colonies vers les anciennes métropoles. Le retour, aujourd'hui, d'une anthropologie exotique mise au service des "natives" (en français nous dirions 
“indigènes") soulève des questions comparables à celles que vous posez pour l'histoire orale des années 1960 . N'y a-t-il pas risque de manipulation, volontaire ou inconsciente, de l'ethnographe par ses indigènes? Comment l'ethnographe choisit-il ceux à qui il "rend" la parole ? Comment préserver la distance scientifique nécessaire, ne pas se contenter d'être le porte-parole, le porte-mémoire, le porte-magnétophone?

\section{Le corpus, I'enquête}

FD: Ce qui est intéressant aujourd'hui, c'est de voir que la technique de l'entretien s'est acclimatée dans toutes les sous-disciplines de l'histoire. L'histoire orale a démarré en histoire sociale et politique dans les années 1970, mais aujourd'hui l'entretien existe partout, en histoire des mentalités, en histoire des techniques, en histoire religieuse, en histoire administrative, en histoire militaire etc. Nous ne somme plus dans la problématique du rapport dominants/dominés, l'entretien est devenu un outil banalisé, ce qui ne veut pas dire pour autant massif et totalement légitimé. Mais attention, actuellement, aucune thèse d'histoire ne se fait qu'à partir de témoignages oraux. Ce qui est possible par exemple aux Etats-Unis est impossible en France.

Sur la question terminologique, nous utilisons effectivement des mots comme corpus d'entretiens ou de témoignages, car pour nous aussi, mais différemment, l'entretien isolé n'a pas de valeur ; il doit précisément se trouver pris dans un réseau documentaire assez vaste d'interrelations et de résonances. Nous parlons de corpus, de collections, de séries parfois, ce qui ne veut pas dire échantillon statistique, même s'il arrive que certains historiens oraux s'essaient à quantifier des données recueillies par entretien qualitatif. Nous considérons que nous ne pouvons guère raisonner à partir d'un seul entretien, mais plutôt à partir d'un ensemble de témoignages construit de manière rigoureuse, qui sera mis à son tour en interrelation avec des documents écrits, voire avec des données d'un autre type, objets, documents photographiques, sources audiovisuelles, presse etc. Il y a là quelque chose d'essentiel: l'historien construit ses sources en réseau, d'emblée et de façon volontairement contradictoire, puisque l'administration de la preuve en histoire résulte de la critique interne et externe des documents, ainsi que du croisement des sources, chaque source 
devant être si possible indépendante les unes des autres. Donc, en définitive, si on veut caricaturer, un témoignage isolé ne vaudra rien en soi. Aujourd'hui, on parle aussi de rotation des points de vue, de "polyphonie". Cette réflexion est le résultat de l'approfondissement méthodologique des années 1980 et 1990.

FW: Pour nous l'unité pertinente n'est pas le "corpus", mais l'enquête, définie par les liens d'interconnaissance entre des enquêtés confrontés à la présence d'un enquêteur. Ce n'est pas en multipliant les entretiens que l'on comprend mieux, c'est en comprenant précisément la signification de cette présence de l'enquêteur pour chacun des enquêtés que l'on peut interpréter un entretien. L'échelle à laquelle doit s'effectuer ce croisement des points de vue est éminemment variable, selon la question traitée et selon le milieu social étudié. Par exemple, comprendre la signification du travail en dehors de l'usine pour les ouvriers d'une usine suppose d'enquêter à l'échelle de la zone de résidence de ces ouvriers. Comprendre les modifications des configurations familiales à l'occasion de l'entrée en dépendance d'un parent âgé suppose de croiser les points de vue de l'ensemble des personnes concernées. Quel que soit leur lieu de résidence, héritiers, obligés alimentaires, aidants professionnels ou non, tout en sachant que l'intégralité des propos recueillis et des comportements observés dépend directement de l'entrée sur le terrain. Comment l'enquêteur est-il entré en contact avec ses différents enquêtés? Les ethnographes travaillent de plus en plus détachés d'une entrée localisée sur le terrain. Ils remontent des réseaux et observent des rencontres. Parler d"'enquête" plutôt que de "corpus", c'est simplement mettre l'accent sur le déroulement chronologique des interactions et sur la place de l'enquêteur.

\section{Témoins, témoignages}

BM: Il m'apparaît que se dessine un accord sur la question de la source, mais il y a peut-être un malentendu sur un autre terme qui me parait important et qui révèle de manière autre les différences entre les deux disciplines, c'est la notion de témoignage, c'est une notion très importante pour l'historien, ça l'est moins pour l'ethnographe.

FW: Pour nous cela n'a aucun sens de penser que nos enquêtés sont des témoins. D'abord on évite toujours de faire des entretiens dit rétrospectifs. 
On a très peu confiance dans la façon dont les gens peuvent raconter le passé et à la limite cela ne nous intéresse pas. Si je reprends le vocabulaire de Marc Bloch, ce qui peut retenir l'ethnographe, c'est l'idée du témoin malgré lui, ce qui nous intéresse c'est ce que disent les gens sans savoir qu'ils sont en train de le dire et cela c'est une position un peu radicale. On ne cherche pas à utiliser ce que les gens disent explicitement, on cherche à retrouver le système de contraintes et de conditions dans lequel sont pris les enquêtés, aussi dans leur relation à l'enquêteur, et pourquoi ils disent cela à un moment donné et pas autre chose: c'est le "pourquoi" et le "comment" (avec quels mots, dans quel ordre) qui nous intéresse, et non pas le "cela". On n'a pas la même lecture du texte de l'entretien.

BM: Il y a une différence sur l'interconnaissance mais il n'y a peutêtre pas non plus la même conception du temps. En particulier lors de la saisie de l'information: le temps est rétrospectif chez les historiens alors que chez les ethnographes l'entretien peut se dérouler dans une durée qui peut être d'une, deux semaines, voir plus d'une année. Je n'ai pas le sentiment que pour les historiens cette durée soit la même.

FD: Sur la question de l'interconnaissance, il me semble que nous nous posons aussi cette question, mais que nous y répondons de manière différente. Nous cherchons notamment à y répondre par l'idée de corpus, en reconstituant un milieu d'interconnaissance assez clos, avec des contours sociaux ou culturels bien définis, bien identifié dans le temps et l'espace: un corps de fonctionnaires, une profession, un métier à une époque donnée, dans un lieu donné. Nous utilisons pour cela des moyens rationalisés (fichiers, annuaires etc.) mais aussi le bouche-à-oreille ou l'effet boule-de-neige.

Pour la question du temps, il y a des différences évidentes. Les historiens travaillent effectivement sur le passé, et la saisie de ce passé à travers le filtre de la mémoire peut apparaitre illusoire ou trompeuse. Evidemment c'est difficile mais on n'abandonne pas. On essaie de suivre ce que dit Marc Bloch, en cherchant à saisir ce que le témoin dit de son passé, la manière dont il le dit, comment et pourquoi, sous quelles contraintes il le dit, et l'on va être particulièrement attentif à ce qu'il dit malgré lui. Il va donc y avoir une double analyse du discours, avec cette idée de rechercher les informations comme des "petites madeleines", de mettre au jour les scarifications de la réalité passée prises dans la mémoire, tout en conservant à l'esprit que tout témoignage est conjugué au présent, 
qu'il est étroitement conditionné par le contexte d'énonciation, le contexte historique d'aujourd'hui. Finalement, nous les historiens oraux, malgré son travail de reconstruction et ses "effets pervers" (Denis Peschanski), nous croyons en la mémoire. C'est une posture optimiste.

Troisième sujet, le témoignage. C'est en effet une figure très ancienne dans la discipline historique, elle est déjà théorisée en partie sous l'Antiquité grecque et aussi par les premiers historiens du christianisme. Cette figure du témoin, qui va ressurgir très brutalement au $\mathrm{xx}^{\mathrm{e}}$ siècle, a peut-être subi une éclipse sous l'Ancien régime, encore que pour l'histoire de l'Eglise, ce soit demeuré important. Ce qui est sûr, c'est que cette notion est liée à une autre notion, qui est l'événement ou le fait (sans connotation positiviste). Le témoin est celui ou celle qui vécu, vu ou entendu, - d'où la notion du témoin oculaire et auriculaire. C'est celui qui possède une connaissance directe de l'événement, qui en a une parcelle de compréhension et qui est prêt à attester de sa réalité: “J’y étais, je l'ai vu, je l'ai entendu, je vous le dis et je vous le confirme". On comprend immédiatement l'importance que cette notion du témoin a pu prendre dans l'histoire du christianisme. Le témoin (martus) est celui qui est prêt à attester de la réalité et de la vérité et à rendre témoignage (martyron) jusqu'à devenir martyr, c'est-à-dire jusqu'à la mort. Et l'on voit cette figure du témoin ressurgir dans le second $x^{\mathrm{e}}$ siècle, dans un tout autre contexte, avec le survivant des camps d'extermination. Le survivant (qui lui aussi est passé par l'épreuve de la mort mais en est revenu) constitue une autre figure de ce témoin de l'extrême qui atteste de la réalité et de la vérité de ce qu'il a vu et vécu, qui en possède une connaissance intime et qui se met, par la parole, au service de cette connaissance.

Voilà pourquoi les historiens n'utilisent guère le terme d'“informateur, car cette posture apparaît comme trop neutre, pas assez impliquée dans l'événement et dans l'attestation de l'événement. Quant au terme "interviewé", ce n'est qu'une indication technique qui signale qu'il y a un magnétophone... Le témoin, oui, c'est finalement le terme qui s'est imposé à l'historien.

FW: Pour réagir à vos propos, je dirais que pour nous c'est l'ethnographe lui-même qui est le témoin et c'est pour cela que nous ne pouvons pas parler de nos enquêtés comme des témoins. Nous allons nous-mêmes sur le terrain et ensuite nous pouvons, et nous demandons à nos collègues de nous faire confiance, nous pouvons dire ce que nous avons observé, 
ce que nous avons vécu, ce que nous avons expérimenté, ce que nous avons vu, etc. Le nœud est en effet très important parce que la place de l'ethnographe dans l'enquête est beaucoup plus centrale et consubstantielle à la discipline que chez vous. J'ai juste une remarque à faire sur la terminologie: la notion d'informateur est un mot utilisé par l'anthropologie classique, aujourd'hui totalement abandonné. En revanche, le terme que nous utilisons est souvent mal compris à l'extérieur de la discipline, c'est le terme d'“indigène". Il veut dire simplement que nous cherchons à comprendre un système de pensée indigène et donc des catégories "émiques" comme disent les Américains, à partir d'un autre système de pensée qui est le nôtre, avec nos catégories “étiques”. Du coup l'ethnographe est celui qui arrive à faire l'expérience de la différence entre son système de pensée à lui ("étique") et le système de pensée indigène ("émique").

Les historiens diraient sans doute "vernaculaire" plutôt qu'indigène. Là aussi c'est un nœud de l'ethnographie: nous considérons que nous sommes capables d'utiliser la distance entre nos propres catégories et les catégories indigènes pour dire, à la fin, des choses à la fois sur les catégories indigènes et sur les nôtres. Cette tension entre l'enquêteur et l'enquêté est à mon avis très spécifique de l'ethnographie. Ce n'est pourtant pas très différent. Lorsque l'enquêteur est très proche socialement et culturellement des enquêtés, il doit convoquer toutes ses connaissances livresques pour établir une distance entre lui et eux, entre lui et lui-même. On pourrait comparer, je pense, la situation de l'ethnographe enquêtant "chez lui" avec la situation des historiens du contemporain: la distance reste à conquérir, la difficulté n'est plus de se familiariser avec un système de pensée différent mais bien de se détacher du sien.

FD: Une remarque encore sur le temps et la durée. En histoire aussi, il y a des collectes de récits de vie qui ont duré plus d'un an et qui sont l'occasion d'importantes interactions interpersonnelles. La constitution d'un corpus de récits de vie dans une institution ou pour un sujet de recherche peut prendre plusieurs années. Alors évidemment, il arrive que l'intervieweur change. Il existe une approche collective possible, une équipe va travailler dans la durée sur un même objet.

BM: La question du témoin qui est au centre des préoccupations des historiens m'incite à poser une question sur la technique même de l'enregistrement de la parole. Il en ressort une autre démarche. Il s'agit bien d'enregistrer des témoignages, de les consigner, nous sommes un peu 
comme dans la situation du tribunal où les témoignages sont des dépositions, donc des déclarations qui doivent être attestées. Ou comme vous venez de le dire en parlant de confession. C'est une posture nouvelle qui n'est plus celle du récit de vie de l'histoire orale, ce n'est pas la même démarche interactive de l'ethnographie...

FW: Vis-à-vis de l'enregistrement, on retrouve la diversité des situations que j'évoquais au début de ce débat. Les ethnographes qui préfèrent l'observation au discours ont tendance à négliger le magnétophone et s'interdisent ainsi tout un travail proche de la sociolinguistique, qui consiste à interroger les mots, les tournures de phrase, les hésitations, les lapsus, les redites... Les ethnographes proches de la sociologie qualitative accumulent des transcriptions d'entretiens, travail ingrat qu'ils délèguent, montrant ainsi qu'ils s'intéressent plus à "ce qui se dit" qu'à “comment c'est dit". Les ethnographes de tradition plus anthropologique, même s'ils utilisent parfois des entretiens enregistrés, sont souvent confrontés à l'impossibilité d'enregistrer, à l'impossibilité de passer du statut d'allié, agissant aux côtés de ses enquêtés et avec eux, au statut de porte-parole, recueillant une parole qu'il cherchera ensuite à préserver et à diffuser. Il faut bien dire que, sur le terrain, nous bricolons: parfois l'entretien ouvrira des portes, et des pistes ; parfois il est inutile de brouiller une relation déjà installée en cherchant à forcer la main pour obtenir... des "discours".

FD: L'enregistrement de la parole par magnétophone répond à plusieurs motivations ou objectifs. Celle du témoin qui trouve moins fatiguant de raconter sa vie ou ses souvenirs de carrière que de les écrire. Celle de l'historien du $\mathrm{xx}^{\mathrm{e}}$ siècle qui répond ainsi aux critiques formulées par les "pères fondateurs" de la discipline historique à l'encontre de la tradition orale et des rumeurs colportées par le bouche-à-oreille: le magnétophone permet de fixer les propos d'un témoin, d'en assurer la transmission, d'en vérifier et d'en confirmer le contenu, d'en étudier la forme et le sens... Celle du chercheur qui trouve bien commode de pouvoir se reporter à l'enregistrement quand ses notes manuscrites s'avèrent insuffisantes. Celle de l'archiviste moderne qui souhaite utiliser les moyens d'expression et de communication de son temps. Celle du public ou de l'auditeur qui se laisse toucher par une voix venue d'outre-tombe.

Il y a aussi, et c'est très fort, cette influence venue du tribunal. Plus que la sphère journalistique que l'on aurait probablement invoquée il y a trente ans, c'est vraiment la référence judiciaire qui s'impose, connotant 
fortement l'utilisation du terme "témoignage" (par exemple il me semble qu'elle est peu valorisée dans des écrits de Marc Bloch, qui a peut-être plus en tête la référence militaire ou même administrative: "faire son rapport"). Il s'est produit comme une contagion, voire comme une contamination. Témoignage des victimes et des bourreaux des crimes de guerre ou des crimes contre l'humanité, témoignages des victimes des guerres civiles ou ethniques, témoignages des victimes et des acteurs de l'apartheid ou des régimes dictatoriaux, témoignages des victimes de l'oppression nazie ou du goulag... Il est d'ailleurs arrivé que, dans certains procès contemporains, les deux figures du témoin, historique et judiciaire, alourdies de façon supplémentaire par leur qualité de parties civiles, se soient trouvées confondues. Sans parler du moment controversé où les historiens ont été convoqués comme "témoins" sous couvert de leur expertise. Il y a là un risque de confusion et de dérive entre l'histoire et la justice des hommes, qui ne manque pas de laisser perplexe.

Enfin, sans aller voir du côté des prétoires, il faut relever l'influence qu'exercent aussi les médias et notamment le genre documentaire historique sur le développement de l'entretien et du témoignage en histoire. Il y a là une pression audiovisuelle qui pèse lourd, tant du point de vue des pratiques que des thématiques ou des périodes étudiées.

On voit ainsi combien en histoire le recours à l'entretien et au témoignage oral se révèle une activité passionnante mais parfois périlleuse.

\section{Ecouter, observer}

FW: J'allais réagir à ce que vous venez de dire en précisant une autre différence: l'observation. Je ne suis pas sûr qu'un historien qui investit tant d'énergie à recueillir le discours de ses témoins en investit autant à observer les lieux où les choses se déroulent, à observer explicitement les pratiques au contact desquelles il se trouve confronté. Par exemple, quand un ethnographe est en entretien et que l'enquêté reçoit un coup de téléphone, évidemment par politesse, il appuie sur le bouton "pause" de son magnétophone. Mais il se met à noter à toute allure ce qui se dit, ou il tente de le mémoriser: ce qui n'est pas censé faire partie de l'entretien est souvent le plus intéressant. Il y a une grande sensibilité à ce qui se passe au moment même de l'entretien, une nécessité à compléter l'entretien par 
l'observation. Pour une raison simple, c'est que nous n'avons aucune ambition à reconstituer un passé et même lorsque les enquêtés évoquent leur passé ce qui nous intéresse c'est ce qu'ils en font aujourd'hui. Il y a là une vraie différence.

FD: L'observation en histoire n'est évidemment en aucun cas prioritaire, puisque notre étude porte par nature sur des faits passés révolus. Il reste qu'en situation d'entretien, nous allons nous aussi porter une attention à l'environnement du témoin, à son attitude et à son comportement, étudier dans quelle situation il nous parle, dans un bureau, dans un café, sur son lieu de travail ou au contraire dans son milieu familial. Tout va être noté, mais noté pour clarifier et qualifier le contexte d'énonciation. C'est un premier point.

Mais il existe aussi des historiens qui savent que l'observation participante ou l'observation directe sont très utiles pour comprendre la population qu'ils étudient, y compris lorsqu'il existe un décalage dans le temps. Prenons l'exemple du travail de Marcel Gillet qui a étudié le monde de la mine au XIx ${ }^{\mathrm{e}}$; il a fait des entretiens de mineurs dans les années cinquante et soixante, très décalés dans le temps par rapport à son objet de recherche initial (Gillet, 1980). Autre exemple: une étudiante en thèse d'histoire sur la direction des Finances extérieures au ministère des Finances dans les années 1945-1955 a effectué un stage d'observation au Service des Affaires internationales de la direction du Trésor en 1994... Dernier exemple: Eric Godelier historien et chercheur en gestion, fait un stage ouvrier pendant un mois chez Usinor dans le cadre de sa thèse consacrée à la formation d'une culture d'entreprise et se livre à une observation directe du milieu ouvrier (Godelier, 2001).

\section{Déléguer}

BM: Je ne veux pas esquiver la question de la délégation, parce qu'il y a là des logiques de l'enquête et des conceptions propres de l'entretien incluses dans un tout. L'entretien n'est qu'une partie dans un tout, ce qu'admet l'historien et il accepte la délégation. En revanche, l'ethnographe semble s'y refuser.

FW: Je distinguerai trois périodes. Première période: la délégation est si naturelle qu'on ne la remarque pas. Le Manuel d'ethnographie de Mauss 
est simplement un manuel de conseils à des enquêteurs auxquels le travail d'enquête est délégué de bout en bout. Cette période se termine avec la professionnalisation des ethnographes, venue du monde anglo-saxon (les Français sont très en retard), et lègue à la discipline une grande méfiance à l'égard de la délégation. Deuxième période: l'ethnographe est d'autant plus arc-bouté contre la délégation du travail de terrain que l'ethnographie est engagée dans sa dérive postmoderne, elle tend à devenir simplement le récit de l'enquête par un ethnographe, celui-ci risque de perdre de vue le fait qu'il travaille sur des gens et pas sur lui-même. Cette période est aujourd'hui presque terminée. Nous sommes restés si sensibles à cette question de la délégation que si, parfois, un ethnographe délègue une partie de son travail, il ne l'avoue pas. Je pense par exemple à Philippe Bourgois, un ethnographe américain d'après la période post-moderne, qui fait beaucoup d'enquêtes lui-même mais, lorsqu'on l'interroge, on s'aperçoit qu'il utilise des enquêteurs en qui il a confiance, qu'il utilise les données qu'ils recueillent, mais il ne l'écrit pas (cf. par exemple, Bourgois, 2000). Derrière la façade du refus de la délégation, il peut y avoir des pratiques de délégation.

Ce qui est important c'est que le moteur de l'enquête ethnographique subsiste, c'est-à-dire qu'on puisse comprendre ce que l'enquêteur a fait, comment s'est nouée la relation d'enquête, et comment le déroulement de l'enquête dit des choses sur ce qui se passe sur le terrain. Nous ne savons pas encore très bien le faire, mais il y a une partie de ces choses-là qui sont délégables à condition que nous sachions repérer quels sont les bons indicateurs pour voir si une relation d'enquête tourne bien ou mal, si l'on est pris dans une "clique" comme disent les ethnographes, une faction qui utilise l'ethnographe contre une autre faction, si l'on est pris dans une relation d'alliance, etc. C'est tout ce déroulement de l'enquête sur lequel nous avons besoin du maximum d'informations pour pouvoir utiliser le matériau "recueilli", en réalité produit, par d'autres. Si nous quittons la posture "mystique" de l'ethnographe vis-à-vis de son terrain, liée peut-être à cette idée de l'ethnographe -témoin ou-martyr, qui a prévalu en anthropologie pendant plusieurs décennies, nous pourrons réfléchir sérieusement à une standardisation de l'enquête ethnographique. Est-ce que le jeu en vaut la chandelle? Certains d'entre nous ne le pensent pas. Le risque d'accumuler, une fois de plus, des matériaux qui se révéleront ensuite inutilisables, est bien présent. 
FD: En histoire, il me semble que la question de la délégation n’a pas été tellement travaillée. Il y a plusieurs situations possibles. Premier cas: l'historien réalise ses propres entretiens, notamment dans le travail de sa thèse ou d'une recherche individuelle, et il n'y a pas de délégation. Deuxième cas: les travaux patrimoniaux collectifs, qui supposent non pas la délégation mais un travail d'équipe. Un chef de projet construit le programme de collecte, construit les problématiques, rédige le canevas d'entretien, choisit une méthode d'entretien, établit un protocole d'interview etc. Ensuite des enquêteurs ou des archivistes oraux réalisent les entretiens qui sont ensuite rassemblés sous forme de collection et déposés dans un lieu patrimonial. Troisième cas: un doctorant ou un chercheur exploite des entretiens réalisés par d'autres et déposés dans un service d'archives. Il n'y a pas à proprement parler de délégation, mais plutôt réutilisation de données collectées et construites par d'autres, selon des procédures qu'il revient au nouvel utilisateur de chercher à connaître.

Il me semble qu'il ne devrait pas y avoir véritablement de gêne théorique sur la question de la délégation, car l'idée domine en histoire que tout document, qu'il soit oral, écrit, audiovisuel, a été "produit", que ses conditions de production doivent être déconstruites, décrites et analysées et qu'il doit être critiqué et évalué. L'important est dans ce contexte de noter et d'enregistrer les éléments qui ont présidé à la production du document (les méta-données) et de les prendre en compte dans l'analyse. Les données concernant l'intervieweur (euse) seront analysées comme les autres et le fait qu'il y ait plusieurs intervieweurs ne gêne pas véritablement l'étude.

Utiliser des entretiens enregistrés par d'autres ne devrait pas non plus théoriquement gêner l'historien, puisque de façon générale, l'historien a l'habitude d'exploiter des sources qui lui sont "extérieures" (archives publiques ou privées, presse, littérature grise etc.). Néanmoins, j’ai pu observer que nombre d'historiens restent réticents à utiliser les archives orales conservées dans les centres patrimoniaux. Les raisons avancées le plus souvent sont de deux ordres: matériel et technique (il faut se déplacer et c'est trop long d'écouter les interviews des autres) ; scientifique (je préfère refaire les interviews moi-même, car étant spécialiste, je saurai mieux interroger le témoin et j'en tirerai de meilleures informations). Il va de soi que ce dernier argument tombe lorsque le témoin n'est plus accessible ou décédé. Les témoignages collectés par d'autres deviennent dès lors plus attractifs. 


\section{Archiver la parole}

BM: Il nous reste peu de temps pour aborder la troisième question sur la patrimonialisation. Pour ma part ce qui m’intéresse ici c'est évidemment la constitution de corpus d'entretiens et leur utilisation non pas aujourd'hui mais dans le futur. Un gros effort a été fait dans la maîtrise de l'enquête de manière à ce que les entretiens soient éventuellement réutilisables. Mais qu'en est-il d'abord pour l'ethnographe?

FW: Pour aller vite, j'aborderai cette question par le biais des pratiques de "revisite". Ces pratiques sont inconnues dans l'anthropologie et dans la sociologie qualitative françaises: elles font peur, elles sont vécues comme un contrôle, comme une intrusion insupportable d'autrui dans une relation "intime" avec son "terrain". Mais aux Etats-Unis, il y a une grande tradition de la revisite, en anthropologie et en sociologie ethnographique. Depuis Oscar Lewis repartant, vingt ou trente ans plus tard, sur le terrain mexicain où avait travaillé Robert Redfield (Lewis, 1963), jusqu'à Michael Burawoy retournant dans le même atelier d'usine où avait travaillé Donald Roy, en passant par Annette Weiner reprenant le terrain trobriandais étudié par Malinowski (Weiner, 1983). De telles expériences, cruciales dans les deux disciplines, n’auraient pas été possibles si les seconds ethnographes n'avaient pu disposer des matériaux accumulés par les premiers. La tradition des "archives" des ethnographes est extrêmement vivante dans le monde anglo-saxon: on peut travailler sur les papiers de Malinowski, de Margaret Mead, de Gregory Bateson... En France, la pénurie d'institutions publiques capables de recueillir les archives scientifiques, jointe au comportement abusif de certains héritiers, nous laisse dans une situation déplorable, qui s'est aggravée tout au long du $\mathrm{Xx}^{\mathrm{e}}$ siècle. Il va de soi que ces papiers sont eux-mêmes révélateurs d'un certain état de la pratique scientifique: notes de terrain ou entretiens enregistrés et transcrits, fichiers informatiques sur des milliers de pages aujourd'hui, milliers de fiches cartonnées autrefois...

Les pratiques de revisite sont triplement passionnantes dans leur rapport à l'histoire: transformations dues à l'histoire de la discipline, transformations dues au changement d'enquêteur, transformations dues à l'histoire des sociétés étudiées. L'anthropologie a mis du temps pour considérer que les sociétés traditionnelles que nous observions étaient historiques, ce n'est pas encore totalement acquis. Par exemple, Annette Weiner 
revisitant, après Malinowski, les îles Trobriand, met l'accent sur le fait que Malinovski avait observé à partir d'un point de vue masculin et que, étant une femme sur le même terrain, elle ne voyait pas les mêmes choses. Cela l'a plus intéressée que les transformations qu'avait subie la société trobriandaise.

FD: Il faudrait de ce point de vue distinguer histoire orale et archives orales. L'histoire orale dans ses débuts n'a envisagé ni la patrimonialisation des entretiens ni leur réutilisation. En revanche, la grande intuition de ceux qui ont théorisé les archives orales (Dominique Aron-Schnapper et ses disciples), c'est précisément de dire que les témoignages oraux, dès lors qu'ils sont organisés en corpus et qu'ils ne sont pas isolés, ont une valeur en soi en tant que traces du passé, qu'ils détiennent des informations précieuses et qu'ils doivent être traités documentairement de manière à pouvoir être réutilisés par les historiens du futur. On ne sait pas aujourd'hui par qui, comment, dans combien de temps, mais on fait le pari qu'ils le seront un jour. On leur accorde une valeur comme traces du passé, mais aussi comme traces du présent (cet horizon temporel qui a décidé l'enregistrement des traces et qui cherche à se souvenir). Il y a donc un jeu à multi temporalité.

En définitive, c'est au chercheur du futur qu'il reviendra de définir, en fonction de son propre questionnement, les apports des témoignages oraux. Evidemment les critères vont changer, ils seront eux-mêmes historicisés. Voilà pourquoi théoriquement nous ne devrions pas être gênés par la revisite, je dirais même que c'est le lot de l'historien que de revisiter des sources qui ont déjà été utilisées et de les faire parler malgré elles et autrement.

Pour certains historiens oraux qui ont constitué leurs propres sources, il peut y avoir des difficultés à s'en détacher. Comment se séparer de quelque chose qui vous est affectivement et intellectuellement très proche ? Seconde question: celle de la confiance. Où déposer en toute sécurité ses enquêtes orales ? C'est un problème auquel les historiens sont très sensibles, parce qu'il s'est produit des erreurs et de pertes de documents. Mais il faut savoir que dans un service patrimonial et historique comme le Service Historique de la Marine, 60\% de l'accroissement des fonds d'archives orales se font par le dépôt d'historiens américains, européens, français qui déposent leurs corpus d'entretiens. Troisièmement, faire en sorte que les enquêtes soient réutilisées, soit, mais par qui et selon quelles règles ? La réflexion est en cours et a démarré depuis peu. Il faut au minimum que chaque dépôt, chaque collection soient rigoureusement et 
précisément documentés, de façon à ce que l'historien qui vient derrière soit capable d'utiliser les données. Il me semble qu'il y a un consensus sur ce point, mais encore faut-il que les pratiques suivent ; il reste beaucoup de travail à faire dans ce domaine des "bonnes pratiques".

FW: Pour les ethnographes, c'est beaucoup plus difficile. Nous avons à peine commencé à réfléchir sur ces questions. Si c'est aussi difficile c'est je crois parce que l'ethnographe considère qu'il a un contrat personnel d'enquête avec ses enquêtés et que ce contrat n'inclus pas la question d'une réutilisation. Dès lors nous mettons surtout l'accent sur la confidentialité, sur l'anonymat, sur le fait que cela ne sortira pas de nos mains et que personne d'autre ne pourra en faire usage. C'est le revers de la médaille: la relation d'enquête ethnographique n'est pas, ou du moins pas toujours, une relation publique ; nous devons protéger nos enquêtés du regard inquisiteur, c'est la condition à laquelle nous pouvons obtenir autre chose qu'une vérité officielle. Mais l'ethnographe engage là sa parole, pour servir d'écran, de médiateur, et ne peut pas envisager sans crainte que son enquête tombe dans le domaine public.

L'histoire orale a fait sienne l'image du témoin, l'ethnographie pourrait se comparer à la confession. Lorsque nous réfléchissons sur notre déontologie professionnelle - pas assez à mon goût -, nous nous sentons proches du secret médical, du secret de la confession. Nous devons prévenir toute utilisation profane de nos matériaux d'enquête. Les historiens sont souvent étonnés de notre manie de l'anonymisation. Côté protecteur, il s'agit de ne rien livrer du secret de l'instruction, pourrions-nous dire ; côté scientifique, nous ne sommes pas intéressés à dire: "cela s'est vraiment passé comme cela", mais bien plutôt à dire: "voici les mécanismes et les processus à l'œuvre dans telle et telle situation sociale". L'indexation spatio-temporelle n'est donc plus nécessaire, une fois que nous l'avons correctement mise en fiches.

FD: L'historien, lui, compte tenu des exigences de sa discipline, a besoin que le témoignage soit rendu public, qu'il puisse être accessible, identifié, vérifié, contrôlé. Plus le témoignage est exploité, plus il est utilisé publiquement et nominativement, finalement mieux c'est. C'est pourquoi bien souvent le pacte d'entretien que l'on essaie d'instaurer avec un témoin, le protocole de communicabilité que l'on met en place dans une campagne d'archives orales vise avant tout à rendre le plus ouvert possible l'accès aux témoignages. Certains formulaires formalisent contractuellement 
les engagements déontologiques des parties et incluent des clauses concernant la publication nominative des témoignages ou leur futur dépôt dans des institutions patrimoniales. Dans le cas des Mémoriaux pour la déportation par exemple, les témoignages recueillis sont destinés à être rendus publics immédiatement et les témoins signent un papier en ce sens.

\section{Ouvrages cités}

BLOCH, Marc. Réflexions d'un historien sur les fausses nouvelles de la guerre. Revue de synthèse historique, vol. 33, p. 13-35, 1921

BOURGOIS, Philippe. En quête de respect: le crack à New-York. Trad. de l'anglais par Lou Aubert. Paris, Seuil, 2001.

BURAWOY, Michael. Global Ethnography: Forces, Connections, and Imaginations in a Postmodern World. Berkeley, University of California Press, 2000.

CRU, Jean-Norton. Témoins. Essais d'analyse et de critique de souvenirs des combattants édités en France de 1955 à 1928. Nancy, Presses universitaires de Nancy, [1929], 1993.

GILLET, Marcel. La sociabilité septentrionale. Archives orales, une autre histoire. Annales ESC, janv.-févr., $\mathrm{n}^{\circ}$ 1: 167-175, 1980.

GODELIER, Eric. De la stratégie locale à la stratégie globale: la formation d'une identité de groupe cher. Usinor 1948-1986. Paris, thèse de doctorat, EHESS, 1995.

LEWIS, Oscar. Les Enfants de Sanchez: autobiographie d'une famille mexicaine. Traduit de l'anglais par Céline Zins. Paris, Gallimard, [1959], 1963.

WEINER, Annette B. La Richesse des femmes ou Comment l'esprit vient aux hommes: îles Trobriand. Traduit de l'américain par Richard Sabban et Danièle Van de Velde. Paris, Éditions du Seuil [1976], 1983.

Résumé: L'entretien oral s'est imposé comme l'un des instruments performants des sciences sociales. Il n'en demeure pas moins que d'une discipline à l'autre, les enjeux théoriques ou pragmatiques ainsi que les conditions de son utilisation varient et parfois se contredisent. Une revue comme la nôtre ouverte aux débats et aux confrontations interdisciplinaires se devait précisément de mettre en évidence quelques-uns de ces enjeux. C'est l'objectif de cet échange entre une historienne, rompue aux techniques de l'entretien et de l'archive orale, et une ethnologue bénéficiant d'une longue expérience de terrain dans laquelle elle a associé observation et entretien. Le débat qui s'est tenu à l'Ecole normale supérieure en juin 2005 a porté sur trois thèmes majeurs: la place de l'entretien dans les traditions disciplinaires, la posture du témoin et la signification du témoignage, les possibilités et les limites de la délégation, les problèmes de l'archivage et du réemploi des enquêtes orales.

Mot-clé: histoire oral; entretien etnographique; temoignage; archivage. 


\section{Arquivos orais e entrevistas etnográficas: um debate entre Florence Des- camps e Florence Weber}

Resumo: A pesquisa oral parece ser um dos instrumentos importantes das ciências sociais. Mas de uma disciplina a outra, os marcos teóricos e pragmáticos, assim como as condições de sua utilização, variam e por vezes se contradizem. $\mathrm{O}$ objetivo deste encontro, entre uma historiadora rompida com as técnicas de entrvista e arquivos orais, e uma etnóloga que se beneficia de uma longa experiência de campo à qual associou a observação e a entrevista, é colocar em evidência alguns destes marcos. O debate, que ocorreu na Escola Normal Superior em junho de 2005, trata de três temas principais : o lugar da entrevista nas tradições disciplinares, a postura da testemunha e a significância do testemunho, e os problemas de arquivamento e utilização das fontes orais.

Palavras-chave: história oral; entrevista etnográfica; testemunho; arquivo oral. 
